Louisiana State University

LSU Digital Commons

$1-1-2019$

\title{
Backstepping design for output feedback stabilization for a class of uncertain systems
}

\author{
Frédéric Mazenc \\ Laboratoire des Signaux et Systèmes \\ Laurent Burlion \\ ONERA - The French Aerospace Lab \\ Michael Malisoff \\ Louisiana State University
}

Follow this and additional works at: https://digitalcommons.Isu.edu/mathematics_pubs

\section{Recommended Citation}

Mazenc, F., Burlion, L., \& Malisoff, M. (2019). Backstepping design for output feedback stabilization for a class of uncertain systems. Systems and Control Letters, 123, 134-143. https://doi.org/10.1016/ j.sysconle.2018.11.007

This Article is brought to you for free and open access by the Department of Mathematics at LSU Digital Commons. It has been accepted for inclusion in Faculty Publications by an authorized administrator of LSU Digital Commons. For more information, please contact ir@lsu.edu. 


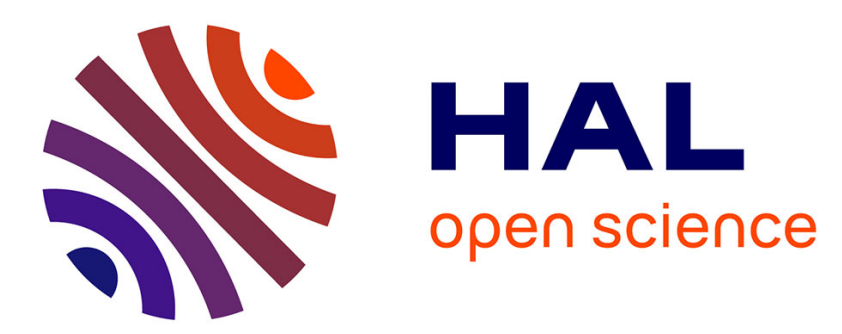

\title{
Backstepping Design for Output Feedback Stabilization for a Class of Uncertain Systems *
}

\author{
Frédéric Mazenc, Laurent Burlion, Michael Malisoff
}

\section{To cite this version:}

Frédéric Mazenc, Laurent Burlion, Michael Malisoff. Backstepping Design for Output Feedback Stabilization for a Class of Uncertain Systems *. Systems and Control Letters, Elsevier, 2019, 10.1016/j.sysconle.2018.11.007 . hal-02342856

\section{HAL Id: hal-02342856 \\ https://hal.inria.fr/hal-02342856}

Submitted on 1 Nov 2019

HAL is a multi-disciplinary open access archive for the deposit and dissemination of scientific research documents, whether they are published or not. The documents may come from teaching and research institutions in France or abroad, or from public or private research centers.
L'archive ouverte pluridisciplinaire HAL, est destinée au dépôt et à la diffusion de documents scientifiques de niveau recherche, publiés ou non, émanant des établissements d'enseignement et de recherche français ou étrangers, des laboratoires publics ou privés. 


\title{
Backstepping Design for Output Feedback Stabilization for a Class of Uncertain Systems*
}

\author{
Frédéric Mazenc ${ }^{\dagger} \quad$ Laurent Burlion ${ }^{\ddagger} \quad$ Michael Malisoff $\S$
}

November 8, 2018

\begin{abstract}
We construct bounded globally asymptotically stabilizing output feedbacks for a family of nonlinear systems, using a dynamic extension and a converging-input-converging-state assumption. We provide sufficient conditions for this assumption to hold, in terms of Lyapunov functions. The novelty is that our construction provides formulas for the control bounds while allowing uncertainties that prevent the use of classical backstepping, and cases where only part of the state variable is available for measurement, without requiring the time lagged states in the feedback control that were required in the artificial delays approach. We illustrate the relevance of our work to engineering in an application to a single-link direct-drive manipulator.
\end{abstract}

\section{Introduction}

Backstepping is a fundamental approach that is often used to construct globally asymptotically stabilizing feedback controls for nonlinear systems having a suitable lower triangular structure called feedback form [5]. Important early contributions to backstepping include [3] and [28], and its numerous applications can be found in [1], [2], [6], [11], [13], [23], [24], [25], and many other works. See also the discussions in the texts and monographs [10] and [12], and many other research articles such as [15].

One challenge in applying traditional backstepping is that it is not always amenable under magnitude constraints on the control laws, which can limit the applicability of classical backstepping or of the bounded backstepping results of [15] and [18]; see [7], [8], [9], [14], [27], and [30] for the importance of control bounds. Another limitation of the approach is due to the fact that in general, it does not apply under outputs, when only a part of the state is measured. Moreover, backstepping generally requires a fictitious feedback that is required to be of class $C^{k}$ when the backstepping is applied $k$ times. Uncertainties in the dynamics or in the output may also be an obstacle (but see [32] for backstepping for certain linear systems that contain uncertainty).

One recent backstepping technique produces controls that contain delayed state observations, even when the current state is available for measurement. This produces delayed state values in the control even when there are no delays in the original systems, so the delays are called artificial. The artificial delays method was introduced in [19], and developed in [20] and [21]. The preceding works do not require $C^{1}$ conditions on the fictitious feedback control. See [16], which used artificial delays to solve a problem involving visual information, where only imprecise measurements of the first backstepping variable are available for use in the control.

The present paper helps to overcome significant remaining challenges in backstepping-based feedback control, by providing globally asymptotically stabilizing feedback controls for a large class of partially linearizable systems; see [10] for discussions on partially linearizable systems. A novel and valuable feature of the present work is its use of a dynamic extension instead of artificial delays, which produces the following advantages as compared to existing backstepping works. First, our new backstepping controls are bounded in situations

\footnotetext{
*Key words: backstepping, nonlinear, output feedback, stabilization. A summary [17] of some of this work appeared in the proceedings of the 2nd IFAC Conference on Modelling, Identification, and Control of Nonlinear Systems; see Section 1 for a comparison with the conference version.

${ }^{\dagger}$ Laboratoire des signaux et systemes, L2S-CNRS-CentraleSupelec, 3 rue Joliot-Curie, 91192 Gif sur Yvette cedex, France frederic.mazenc@12s.centralesupelec.fr.

¥Onera - The French Aerospace Lab, F-31055 Toulouse, France, Laurent.Burlion@onera.fr.

$\S$ Department of Mathematics, Louisiana State University, Baton Rouge, LA 70803-4918, USA, malisoff@lsu.edu.
} 
where bounded feedbacks can be expected (which contrasts with [22] where the controls obtained for the original systems are not bounded controls), and our method produces closed form expressions for the control bounds. Second, our new method of this work ensures global asymptotic stabilization in cases where the system from which the backstepping begins contains uncertainties, rather than the weaker condition of input-to-state stability with respect to uncertainties in the system that was provided in works such as [21]. Finally, this work applies in cases where only a part of the state variable is measured and where some parts of the dynamics are not known with accuracy, while still allowing cases where the fictitious feedback is not necessarily $C^{1}$.

This work is organized as follows. Our main theorem is shown in Section 2. Section 3 provides methods to verify that our assumptions from Section 2 are satisfied. An illustration based on a direct drive manipulator and an additional example are given in Section 4, and Section 5 summarizes the value of our paper and our suggestions for future research. This article adds value as compared with our preliminary conference version [17] by (i) providing explicit formulas for our control bounds in our theorem, (ii) showing how the converging-inputconverging-state assumption of our theorem can be satisfied with fictitious feedbacks that are not necessarily $C^{1}$, and (iii) analyzing the effects of multiple uncertain constants in the manipulator illustration. These three features were not contained in [17], which required the fictitious feedbacks to be $C^{1}$ in its sufficient conditions, did not provide formulas for the control bounds, and only allowed one unknown constant in its one illustration.

Our notation will be simplified whenever no confusion would arise from the context. The dimensions of our Euclidean spaces are arbitrary unless we indicate otherwise. The Euclidean norm in $\mathbb{R}^{a}$, and the induced norm of matrices, are both denoted by $|\cdot|$, and $|\cdot|_{\infty}$ is the usual sup norm. For each constant $J>0$, we let sat denote the symmetric saturation function that is defined by $\operatorname{sat}_{J}(x)=\max \{-J, \min \{J, x\}\}$ for all $x \in \mathbb{R}$, where $J$ is called the saturation level. Finally, we use the usual classes of comparison functions $\mathcal{K} \mathcal{L}$ and $\mathcal{K}_{\infty}$ from [12].

\section{Main Theorem}

\subsection{The studied system}

We consider the nonlinear time-varying system

$$
\left\{\begin{aligned}
\dot{x} & =f\left(t, x, g_{1}\right) \\
\dot{g}_{1} & =a_{1,1} g_{1}+a_{1,2} g_{2} \\
\dot{g}_{2} & =a_{2,1} g_{1}+a_{2,2} g_{2}+a_{2,3} g_{3} \\
\vdots & \\
\dot{g}_{n} & =a_{n, 1} g_{1}+a_{n, 2} g_{2}+\cdots+a_{n, n} g_{n}+u+\Omega(\mathcal{Y}(t, x), G)
\end{aligned}\right.
$$

where $x$ is valued in $\mathbb{R}^{p}$, the state components $G=\left(g_{1}, \ldots, g_{n}\right)$ are valued in $\mathbb{R}^{n}, f$ is locally Lipschitz with respect to $\left(x, g_{1}\right)$ and piecewise continuous and locally bounded with respect to $t, u$ is the scalar-valued input, $\mathcal{Y}: \mathbb{R} \times \mathbb{R}^{p} \rightarrow \mathbb{R}^{l}$ is a continuous function, each $a_{i, j} \in \mathbb{R}$ is a constant, $a_{i, i+1} \neq 0$ holds for $i=1,2 \ldots n-1$ (with no sign constraint on the $a_{i j}$ 's), and $\Omega$ is a locally Lipschitz function that is bounded by a known constant $\bar{\Omega} \geq 0$. Systems of this type result from partial linearization and are frequently encountered in practice.

In this section, we assume that the $\mathbb{R}^{l+n}$ valued output is

$$
Y(t)=\left(\mathcal{Y}(t, x(t)), g_{1}(t), \ldots, g_{n}(t)\right)^{\top},
$$

which is realistic in practice. We next state our main assumption; see Section 3 for Lyapunov function based sufficient conditions for this assumption to hold.

Assumption 1. There exist a locally Lipschitz scalar valued function $\psi$ that is bounded by a known constant $\bar{\psi} \geq 0$, and a constant $k>0$, such that for any continuous function $d:[0,+\infty) \rightarrow \mathbb{R}$ that converges exponentially to the origin, all solutions $\left(\xi, \lambda_{1}, \ldots, \lambda_{n}\right):[0,+\infty) \rightarrow \mathbb{R}^{p+n}$ of

$$
\left\{\begin{aligned}
\dot{\xi} & =f\left(t, \xi, \lambda_{1}+d(t)\right) \\
\dot{\lambda}_{1} & =k\left[-\lambda_{1}+\lambda_{2}\right] \\
\vdots & \\
\dot{\lambda}_{n} & =k\left[-\lambda_{n}+\psi(t, \mathcal{Y}(t, \xi))\right]
\end{aligned}\right.
$$

converge to the origin as $t \rightarrow+\infty$. 
In terms of the matrices

$$
A=\left[\begin{array}{ccccc}
a_{1,1} & a_{1,2} & 0 & \ldots & 0 \\
a_{2,1} & a_{2,2} & a_{2,3} & \ldots & 0 \\
\vdots & & \ddots & \ddots & \vdots \\
\vdots & & & \ddots & a_{n-1, n} \\
a_{n, 1} & a_{n, 2} & \ldots & \ldots & a_{n, n}
\end{array}\right] \in \mathbb{R}^{n \times n}
$$

for $n>1$ and $A=a_{1,1}$ for $n=1$ and $B=(0,0, \ldots, 1)^{\top} \in \mathbb{R}^{n}$, our second assumption is:

Assumption 2. There is a locally Lipschitz function $\varpi: \mathbb{R}^{n} \rightarrow \mathbb{R}$ that is bounded by a known constant $\bar{\varpi} \geq 0$ such that the origin of the system

$$
\dot{\Gamma}(t)=A \Gamma(t)+B \varpi(\Gamma(t))
$$

is a globally asymptotically and locally exponentially stable equilibrium.

See [26] for general conditions under which Assumption 2 is satisfied, in terms of the eigenvalues of $A$.

\subsection{Statement of and discussion on theorem}

To state our theorem, we first recursively define the following real constants, in terms of the constant $k$ from Assumption 1 and the $a_{i, j}$ 's from the system dynamics (1) and (4). We set

$$
\begin{gathered}
c_{1}=-\sum_{i=1}^{n} a_{n, i} l_{i, 1}-k l_{n, 1} \text {, and } c_{j}=-\sum_{i=j}^{n} a_{n, i} l_{i, j}-k l_{n, j}+k l_{n, j-1} \text { if } 2 \leq j \leq n \text {, where } \\
l_{1,1}=-1 ; \quad l_{2,1}=\frac{a_{1,1}+k}{a_{1,2}} ; \quad l_{j, j}=\frac{k}{a_{j-1, j}} l_{j-1, j-1} \text { if } 2 \leq j \leq n ; \\
l_{j, 1}=-\sum_{s=1}^{j-1} \frac{a_{j-1, s}}{a_{j-1, j}} l_{s, 1}-\frac{k}{a_{j-1, j}} l_{j-1,1} \text { if } 3 \leq j \leq n ; \quad l_{j, w}=0 \text { if } j<w \leq n ; \\
\text { and } l_{j, w}=-\sum_{s=w}^{j-1} \frac{a_{j-1, s}}{a_{j-1, j}} l_{s, w}-\frac{k}{a_{j-1, j}} l_{j-1, w}+\frac{k}{a_{j-1, j}} l_{j-1, w-1} \text { if } 3 \leq j \leq n \text { and } 2 \leq w \leq j-1 .
\end{gathered}
$$

Formulas (6b)-(6d) define a lower triangular matrix $L=\left[l_{i, j}\right] \in \mathbb{R}^{n \times n}$. We are ready to state and prove the following result, where sat was defined in the previous section:

Theorem 1. Let the system (1) satisfy Assumptions 1-2, let $\epsilon$ be any positive constant, choose the constants $l_{i, j}$ and $c_{j}$ from (6a)-(6d), and choose the $n \times n$ matrix $L=\left[l_{i, j}\right]$. Then all solutions $(x, G):[0,+\infty) \rightarrow \mathbb{R}^{p+n}$ of (1), in closed loop with the dynamic output feedback

$$
\begin{aligned}
u(G, Z, x) & =-\operatorname{sat}_{\bar{Z}}\left(\sum_{j=1}^{n} c_{j} z_{j}+k l_{n, n} \psi(t, \mathcal{Y}(t, x))\right)+\varpi(G+L Z)-\Omega(\mathcal{Y}(t, x), G) \\
\dot{z}_{1} & =k\left[-z_{1}+z_{2}\right] \\
\vdots & \\
\dot{z}_{n-1} & =k\left[-z_{n-1}+z_{n}\right] \\
\dot{z}_{n} & =k\left[-z_{n}+\psi(t, \mathcal{Y}(t, x))\right]
\end{aligned}
$$

with $Z=\left(z_{1}, \ldots, z_{n}\right)$ and the saturation level

$$
\bar{Z}=\bar{\psi}(1+\epsilon)\left(\sum_{j=1}^{n}\left|c_{j}\right|+k\left|\ell_{n, n}\right|\right),
$$

are such that $\lim _{t \rightarrow+\infty}(x(t), G(t))=0$. Also, the feedback $u$ in (7) is bounded by the constant $\bar{Z}+\bar{\varpi}+\bar{\Omega}$.

Before turning to the proof of Theorem 1, we make several remarks on its motivation and value.

1) Our assumptions do not ensure forward completeness of the closed loop $(x, G)$ dynamics from our theorem, but the theorem is true without such forward completeness because it only applies to solutions that are defined 
on $[0,+\infty)$. In particular, it allows finite time escape phenomena. On the other hand, we can ensure forward completeness of this closed loop system under additional linear growth conditions on $f, \psi$, and $\mathcal{Y}$ in the state. 2) The formula (7) for the control law does not incorporate the first derivative of $\psi(t, \mathcal{Y}(t, x(t)))$, which plays the role of the fictitious control of the classical backstepping approach. Hence, it applies even when $\psi$ is not of class $C^{1}$. While the works $[21,22]$ also did not require the fictitious feedback $\psi$ to be $C^{1}$, these previous works use a very different artificial delay approach that did not allow outputs and they required full knowledge of the nonlinear subsystem to ensure uniform global asymptotic stability of the zero equilibrium.

3) One can use changes of variables and a feedback to transform (1) into a system of the form (3) with $d=0$ whose exponential stability property is ensured by Assumption 1. However, such a result would not be satisfactory, because the feedback obtained that way may possess inappropriate properties. For instance, in general it would be unbounded. This motivates our alternative approach, based on dynamic extensions.

4) None of our assumptions imply that $f$ has to be known with accuracy. Also, we only require measurements of $\psi(t, \mathcal{Y}(t, x))$ and $\Omega(\mathcal{Y}(t, x), G)$ in the control, instead of $\mathcal{Y}$ itself. This is a weaker requirement than requiring measurements of $\mathcal{Y}(t, x)$ or $G$, because the $\psi$ allows uncertainties in the measurements, and because both $\psi$ and $\Omega$ allow cases where only some (instead of all) components of $\mathcal{Y}$ are available for measurements, which can occur in autonomous vehicle/robotic guidance, navigation and control applications with unknown states.

\subsection{Proof of Theorem 1}

An important feature of the proof consists of making $g_{1}(t)-z_{1}(t)$ converge to zero, instead of the classical backstepping approach of making $g_{1}(t)-\psi(t, \mathcal{Y}(t, x(t)))$ converge to zero. To achieve our goal, we use the constants $l_{i, j}$ from (6b)-(6d) to make the change of coordinates $R=G+L Z$. We first assume that $n>1$. Later we explain what occurs when $n=1$.

Induction assumption: We prove the following by induction on $i$ : For all $i \in\{2, \ldots, n\}$, we have

$$
\left\{\begin{aligned}
\dot{r}_{1} & =a_{1,1} r_{1}+a_{1,2} r_{2} \\
\dot{r}_{2} & =a_{2,1} r_{1}+a_{2,2} r_{2}+a_{2,3} r_{3} \\
\vdots & \\
\dot{r}_{i-1} & =a_{i-1,1} r_{1}+a_{i-1,2} r_{2}+\cdots+a_{i-1, i} r_{i}
\end{aligned}\right.
$$

for all $t \geq 0$.

First step: $i=2$. Since $r_{1}=g_{1}-z_{1}$, we have

$$
\dot{r}_{1}=a_{1,1} g_{1}+a_{1,2} g_{2}-k\left[-z_{1}+z_{2}\right]=a_{1,1} r_{1}+a_{1,2}\left[g_{2}+\frac{a_{1,1}+k}{a_{1,2}} z_{1}-\frac{k}{a_{1,2}} z_{2}\right] .
$$

Since our formulas $(6 \mathrm{~b})$ give

$$
r_{2}=g_{2}+l_{2,1} z_{1}+l_{2,2} z_{2}=g_{2}+\frac{a_{1,1}+k}{a_{1,2}} z_{1}-\frac{k}{a_{1,2}} z_{2},
$$

we obtain $\dot{r}_{1}=a_{1,1} r_{1}+a_{1,2} r_{2}$. Thus the induction assumption is satisfied at the first step.

Step $i$ : Assume that the induction assumption is satisfied at a step $i \in\{2, \ldots, n-1\}$. Then since

$$
r_{j}=g_{j}+\sum_{m=1}^{j} l_{j, m} z_{m}
$$

holds for $j=1,2, \ldots, i$, we have

$$
\begin{aligned}
\dot{r}_{i}= & a_{i, 1} g_{1}+a_{i, 2} g_{2}+\ldots+a_{i, i+1} g_{i+1}+\sum_{m=1}^{i} l_{i, m} k\left[-z_{m}+z_{m+1}\right] \\
= & a_{i, 1} r_{1}+a_{i, 2} r_{2}+\ldots+a_{i, i} r_{i}+a_{i, i+1} g_{i+1}+a_{i, 1} z_{1}-a_{i, 2}\left(l_{2,1} z_{1}+l_{2,2} z_{2}\right)-\ldots \\
& -a_{i, i} \sum_{m=1}^{i} l_{i, m} z_{m}+\sum_{m=1}^{i} l_{i, m} k\left[-z_{m}+z_{m+1}\right] .
\end{aligned}
$$

Thus, since our recursive formulas from (6b)-(6d) (with the choice $j=i+1$ ) also give

$$
r_{i+1}=g_{i+1}+\frac{a_{i, 1}}{a_{i, i+1}} z_{1}-\frac{a_{i, 2}}{a_{i, i+1}}\left(l_{2,1} z_{1}+l_{2,2} z_{2}\right)-\ldots-\frac{a_{i, i}}{a_{i, i+1}} \sum_{m=1}^{i} l_{i, m} z_{m}+\frac{1}{a_{i, i+1}} \sum_{m=1}^{i} l_{i, m} k\left[-z_{m}+z_{m+1}\right]
$$


we can multiply both sides of (13) by $a_{i, i+1}$ and then combine (12)-(13) to obtain

$$
\dot{r}_{i}=a_{i, 1} r_{1}+a_{i, 2} r_{2}+\ldots+a_{i, i+1} r_{i+1},
$$

so the induction assumption is satisfied at the step $i+1$.

Computing the time derivative of $r_{n}=g_{n}+l_{n, 1} z_{1}+\ldots+l_{n, n} z_{n}$, it follows from our formulas for the $c_{j}$ 's in (6a) that the system (1) can be written as

$$
\left\{\begin{aligned}
\dot{x} & =f\left(t, x, z_{1}+r_{1}\right) \\
\dot{r}_{1} & =a_{1,1} r_{1}+a_{1,2} r_{2} \\
\dot{r}_{2} & =a_{2,1} r_{1}+a_{2,2} r_{2}+a_{2,3} r_{3} \\
\vdots & \\
\dot{r}_{n} & =a_{n, 1} r_{1}+a_{n, 2} r_{2}+\cdots+a_{n, n} r_{n}+u+\sum_{j=1}^{n} c_{j} z_{j}+k l_{n, n} \psi(t, \mathcal{Y}(t, x))+\Omega(\mathcal{Y}(t, x), G) .
\end{aligned}\right.
$$

If instead $n=1$, then we again obtain (15) with only the dynamics for $x$ and $r_{n}$ present. Then the closed-loop system from the statement of our theorem is

$$
\left\{\begin{aligned}
\dot{x} & =f\left(t, x, z_{1}+r_{1}\right) \\
\dot{R} & =A R+B\left[\varpi(R)-\operatorname{sat}_{\bar{Z}}\left(\sum_{j=1}^{n} c_{j} z_{j}+k l_{n, n} \psi(t, \mathcal{Y}(t, x))\right)+\sum_{j=1}^{n} c_{j} z_{j}+k l_{n, n} \psi(t, \mathcal{Y}(t, x))\right] \\
\dot{z}_{1} & =k\left[-z_{1}+z_{2}\right] \\
\vdots & \\
\dot{z}_{n} & =k\left[-z_{n}+\psi(t, \mathcal{Y}(t, x))\right] .
\end{aligned}\right.
$$

Consider any solution $(x, R, z):[0,+\infty) \rightarrow \mathbb{R}^{p+2 n}$ of $(16)$.

Since Assumption 1 ensures that $\psi$ is bounded by $\bar{\psi}$, it follows from (16) that there is a finite value $t_{a} \geq 0$ such that for all $t \geq t_{a}$, the inequality

$$
\left|\sum_{j=1}^{n} c_{j} z_{j}(t)+k l_{n, n} \psi(t, \mathcal{Y}(t, x(t)))\right| \leq \bar{Z}
$$

is satisfied. Hence, when $t \geq t_{a}$, the closed-loop system is

$$
\left\{\begin{aligned}
\dot{x} & =f\left(t, x, z_{1}+r_{1}\right) \\
\dot{R} & =A R+B \varpi(R) \\
\dot{z}_{1} & =k\left[-z_{1}+z_{2}\right] \\
\vdots & \\
\dot{z}_{n} & =k\left[-z_{n}+\psi(t, \mathcal{Y}(t, x))\right]
\end{aligned}\right.
$$

Assumption 2 ensures that the $R$-subsystem of the system (18) is globally asymptotically and locally exponentially stable. Then Assumption 1 allows us to conclude.

\section{Lemma for Checking Assumptions}

Assumption 2 is a simple classical assumption that can often be checked easily [26]; see our illustrations below. However, checking Assumption 1 can be nontrivial. Our work [17] provided sufficient conditions for Assumption 1 to hold, under the assumption that the fictitious feedback $\psi$ is $C^{1}$. In this section, we provide an alternative method that allows the fictitious feedback $\psi$ to be Lipschitz but not necessarily $C^{1}$.

We consider the system

$$
\left\{\begin{aligned}
\dot{x} & =f\left(t, x, \lambda_{1}+d(t)\right) \\
\dot{\lambda}_{i} & =k\left[-\lambda_{i}+\lambda_{i+1}\right], \text { if } 1 \leq i \leq n-1 \\
\dot{\lambda}_{n} & =k\left[-\lambda_{n}+\psi(t, x)\right]
\end{aligned}\right.
$$


where the constant $k>0$ will be further restricted below, and the continuous function $d:[0,+\infty) \rightarrow \mathbb{R}$ exponentially converges to 0 . Then (19) is a special case of (3). Our lemma will assume the following three assumptions, where the second assumption is a variant of the standard input-to-state stability Lyapunov function decay condition, and where the third one becomes a linear growth condition on $f$ in the significant special case where $W$ is a quadratic (but see Remark 3.1 for a way to relax the uniform global Lipschitzness requirement from Assumption 3 so that we instead only assume a uniform local Lipschitzness condition on $\psi$ ):

Assumption 3. There is a constant $q_{1}>0$ such that $|\psi(t, a)-\psi(t, b)| \leq q_{1}|a-b|$ holds for all $a$ and $b$ in $\mathbb{R}^{p}$ and all $t \geq 0$, and the function $f$ is such that (19) is forward complete. Also, $\psi(t, 0)=0$ for all $t \geq 0$.

Assumption 4. There exist a $C^{1}$ function $V$, a uniformly continuous positive definite function $W$, a constant $\mathcal{L}>0$, and class $\mathcal{K}_{\infty}$ functions $\gamma_{1}$ and $\gamma_{2}$ such that $\gamma_{1}(|x|) \leq V(t, x) \leq \gamma_{2}(|x|)$ hold for all $(t, x) \in[0,+\infty) \times \mathbb{R}^{p}$ and such that the time derivative of $V$ along all solutions of $\dot{x}(t)=f(t, x(t), \psi(t, x(t))+\delta(t))$ for all choices of the continuous function $\delta$ satisfies $\dot{V}(t) \leq-W(x(t))+\mathcal{L}|\delta(t)|^{2}$ for all $t \geq 0$.

Assumption 5. There are constants $q_{2}>0$ and $q_{3}>0$ such that $f$ from (19) satisfies

$$
|f(t, x, \psi(t, x)+\Delta)|^{2} \leq q_{2} W(x)+q_{3} \Delta^{2}
$$

for all $t \geq 0, x \in \mathbb{R}^{p}$, and $\Delta \in \mathbb{R}$, where $W$ is from Assumption 4 .

We emphasize that the requirement (20) will be checkable in practice, because $W$ will be known from the decay estimate from Assumption 4; see the examples below. We now prove:

Lemma 1. Let Assumptions 3-5 hold, and choose any constants $\beta \in(0,1)$ and $k>0$ such that

$$
\begin{aligned}
& 2 e^{2}(n-1) \beta<e-1 \text { and } k>\max \left\{\bar{k}_{a}, \bar{k}_{b}\right\} \text {, where } \\
& \bar{k}_{a}=q_{1} e \sqrt{\frac{2 n q_{3} \beta\left(\beta^{-n}-1\right)}{\left[e-1-2 e^{2}(n-1) \beta\right](1-\beta)}} \text { and } \bar{k}_{b}=\sqrt{\frac{n q_{1}^{2} q_{2} e^{3 / 2}\left(\beta^{-n}-1\right) \mathcal{L}}{(e-1)(\sqrt{e}-1)\left(\beta^{-1}-1\right)}} .
\end{aligned}
$$

Then all solutions $(x, \lambda):[0,+\infty) \rightarrow \mathbb{R}^{p+n}$ of (19) converge to 0 as $t \rightarrow+\infty$.

Proof. To make the proof easy to follow, we first summarize the main ideas of the proof, before providing all needed technical details. In the first part of the proof, we prove an input-to-state stability type decay condition on a suitable nonnegative valued function $\kappa$ of the differences $\lambda_{i}(t)-\lambda_{i+1}(t)$ of the states of the $\lambda$ subsystem of (19), with an overshoot depending on the function $d^{2}$ and $W(x(t))$. Then, we use Assumption 4 to ensure that $x(t)$ and so also $\lambda(t)$ converge to zero as $t \rightarrow+\infty$, using the decay condition from the first part of the proof.

We now provide the needed technical details. Fix any solution $(x, \lambda):[0,+\infty) \rightarrow \mathbb{R}^{p+n}$ of (19). For the rest of the proof, we write $\psi(t)$ to mean $\psi(t, x(t))$. Set $\rho_{i}(t)=\lambda_{i}(t)-\lambda_{i+1}(t)$ for $i=1,2, \ldots, n-1$, $\rho_{n}(t)=\lambda_{n}(t)-\psi(t)$, and $h=\frac{1}{k}$. Then we can apply variation of parameters to the $\lambda_{n}$ subsystem of (19) to get

$$
\begin{aligned}
\rho_{n}(t) & =e^{-1} \lambda_{n}(t-h)+k \int_{t-h}^{t} e^{-k(t-m)} \psi(m) \mathrm{d} m-\psi(t) \\
& =e^{-1} \rho_{n}(t-h)+e^{-1}[\psi(t-h)-\psi(t)]+k \int_{t-h}^{t} e^{k(m-t)}[\psi(m)-\psi(t)] \mathrm{d} m,
\end{aligned}
$$

by writing $\psi(m)$ as $[\psi(m)-\psi(t)]+\psi(t)$ in the first integral in (22). Here and in the sequel, all equalities and inequalities should be understood to hold for all $t \geq h$ along our arbitrary chosen solution of (19).

Moreover, for $i=1$ to $n-1$, we can use the preceding argument with $\rho_{n}$ and $\psi$ replaced by $\rho_{i}$ and $\lambda_{i+1}$ respectively and the fact that $\dot{\lambda}_{i+1}(t)=-k \rho_{i+1}(t)$ to obtain

$$
\begin{aligned}
\rho_{i}(t) & =e^{-1} \rho_{i}(t-h)+e^{-1}\left[\lambda_{i+1}(t-h)-\lambda_{i+1}(t)\right]+k \int_{t-h}^{t} e^{k(m-t)}\left[\lambda_{i+1}(m)-\lambda_{i+1}(t)\right] \mathrm{d} m \\
& =e^{-1} \rho_{i}(t-h)+k e^{-1} \int_{t-h}^{t} \rho_{i+1}(r) \mathrm{d} r+k^{2} \int_{t-h}^{t} e^{k(m-t)} \int_{m}^{t} \rho_{i+1}(r) \mathrm{d} r \mathrm{~d} m,
\end{aligned}
$$

where the last equality used the Fundamental Theorem of Calculus to express $\lambda_{i+1}(t-h)-\lambda_{i+1}(t)$ and $\lambda_{i+1}(m)-$ $\lambda_{i+1}(t)$ as integrals of $-k \rho_{i+1}$ over $[t-h, t]$ and $[m, t]$ respectively. Let $\varpi_{i}(t)=\left|\rho_{i}(t)\right|$. Then, for $i=1$ to $n-1$, we can use (23) to obtain

$$
\varpi_{i}(t) \leq e^{-1} \varpi_{i}(t-h)+\left[k e^{-1}+k^{2} \int_{-h}^{0} e^{k m} \mathrm{~d} m\right] \int_{t-h}^{t} \varpi_{i+1}(r) \mathrm{d} r=e^{-1} \varpi_{i}(t-h)+k \int_{t-h}^{t} \varpi_{i+1}(r) \mathrm{d} r,
$$


by computing the absolute values of both sides of (23), then applying the triangle inequality to the resulting right side, and finally using the bound $\left|\int_{m}^{t} \rho_{i+1}(r) \mathrm{d} r\right| \leq \int_{t-h}^{t} \varpi_{i+1}(r) \mathrm{d} r$ for all $m \in[t-h, t]$. It follows from Young's and Jensen's inequalities that, for any constant $p>0$, the inequality

$$
\varpi_{i}^{2}(t) \leq(1+p) e^{-2} \varpi_{i}^{2}(t-h)+\left(1+\frac{1}{p}\right) k^{2} h \int_{t-h}^{t} \varpi_{i+1}^{2}(r) \mathrm{d} r
$$

is satisfied if $1 \leq i \leq n-1$, by using the relation $(a+b)^{2} \leq(1+p) a^{2}+(1+1 / p) b^{2}$ where $a$ and $b$ are the second to last and last terms in (24), respectively. Similarly, it follows from applying the upper bound

$$
\sup _{t-h \leq m \leq t}|\psi(m)-\psi(t)| \leq q_{1} \int_{t-h}^{t}|\dot{x}(r)| \mathrm{d} r
$$

twice to the last terms in (22) (where (26) used the Lipschitz constant $q_{1}$ from Assumption 3), and then using Jensen's inequality in the result, that for any constant $p>0$,

$$
\varpi_{n}^{2}(t) \leq(1+p) e^{-2} \varpi_{n}^{2}(t-h)+\left(1+\frac{1}{p}\right) q_{1}^{2} h \int_{t-h}^{t}|\dot{x}(r)|^{2} \mathrm{~d} r .
$$

Let us use the simplifying common notation $\mu_{i}(t)=\varpi_{i}^{2}(t)$ for $i=1$ to $n$ and set $p=e-1$. Then

$$
\begin{aligned}
& \mu_{i}(t) \leq e^{-1} \mu_{i}(t-h)+\left(1+\frac{1}{p}\right) k^{2} h \int_{t-h}^{t} \mu_{i+1}(r) \mathrm{d} r, \quad 1 \leq i \leq n-1 \text { and } \\
& \mu_{n}(t) \leq e^{-1} \mu_{n}(t-h)+\left(1+\frac{1}{p}\right) q_{1}^{2} h \int_{t-h}^{t}|\dot{x}(r)|^{2} \mathrm{~d} r .
\end{aligned}
$$

Set

$$
\varsigma(t)=\sum_{j=1}^{n} \beta^{n-j} \mu_{j}(t) \text { and } \kappa\left(\varsigma_{t}\right)=\int_{t-h}^{t} e^{-b_{1}(t-r)} \varsigma(r) \mathrm{d} r, \text { where } b_{1}=\frac{1}{2 h} .
$$

Then it follows from using (28) to upper bound the terms being summed in (29), and then using the inequalities $\mu_{j+1}(t) \leq \beta^{j+1-n} \varsigma(t)$ for $j=0$ to $n-1$, that

$$
\begin{aligned}
\varsigma(t) & \leq \sum_{j=1}^{n-1} \beta^{n-j}\left[e^{-1} \mu_{j}(t-h)+\left(1+\frac{1}{p}\right) k^{2} h \int_{t-h}^{t} \mu_{j+1}(r) \mathrm{d} r\right]+e^{-1} \mu_{n}(t-h)+\left(1+\frac{1}{p}\right) q_{1}^{2} h \int_{t-h}^{t}|\dot{x}(r)|^{2} \mathrm{~d} r \\
& \leq e^{-1} \varsigma(t-h)+\sum_{j=1}^{n-1} \beta^{n-j}\left[\left(1+\frac{1}{p}\right) k^{2} h \int_{t-h}^{t} \beta^{j-n+1} \varsigma(r) \mathrm{d} r\right]+\left(1+\frac{1}{p}\right) q_{1}^{2} h \int_{t-h}^{t}|\dot{x}(r)|^{2} \mathrm{~d} r \\
& \leq e^{-1} \varsigma(t-h)+(n-1) \frac{e k^{2}}{e-1} h \beta \int_{t-h}^{t} \varsigma(r) \mathrm{d} r+\frac{e}{e-1} q_{1}^{2} h \int_{t-h}^{t}\left|f\left(r, x(r), \psi(r)+\sum_{l=1}^{n} \rho_{l}(r)+d(r)\right)\right|^{2} \mathrm{~d} r,
\end{aligned}
$$

where the last inequality followed because $\sum_{l=1}^{n} \rho_{l}(t)=\lambda_{1}(t)-\psi(t)$.

By applying Assumption 5 to the function $\Delta(r)=\sum_{\ell=1}^{n} \rho_{\ell}(r)+d(r)$, it follows that

$$
\varsigma(t) \leq e^{-1} \varsigma(t-h)+\frac{(n-1) h \beta e k^{2}}{e-1} \int_{t-h}^{t} \varsigma(r) \mathrm{d} r+\frac{q_{4} h e}{e-1} \int_{t-h}^{t} W(x(r)) \mathrm{d} r+\frac{q_{1}^{2} q_{3} h e}{e-1} \int_{t-h}^{t}\left(\sum_{l=1}^{n} \rho_{l}(r)+d(r)\right)^{2} \mathrm{~d} r,
$$

where $q_{4}=q_{1}^{2} q_{2}$. Then for any constant $\epsilon_{0} \in(0,1)$, we can use the inequality $(a+b)^{2} \leq\left(1+\epsilon_{0}\right) a^{2}+\left(1+\left(1 / \epsilon_{0}\right)\right) b^{2}$ for suitable nonnegative values $a$ and $b$ to find a constant $\bar{d}>0$ (depending on $\epsilon_{0}, k$, and $n$ ) such that

$$
\begin{aligned}
\varsigma(t) & \leq e^{-1} \varsigma(t-h)+\frac{e k^{2}(n-1)}{e-1} h \beta \int_{t-h}^{t} \varsigma(r) \mathrm{d} r+\frac{e q_{4} h}{e-1} \int_{t-h}^{t} W(x(r)) \mathrm{d} r+\frac{\left(1+\epsilon_{0}\right) e}{e-1} q_{1}^{2} q_{3} h \int_{t-h}^{t}\left(\sum_{l=1}^{n} \rho_{l}(r)\right)^{2} \mathrm{~d} r+d_{*}(t) \\
& \leq e^{-1} \varsigma(t-h)+\frac{e k^{2}(n-1)}{e-1} h \beta \int_{t-h}^{t} \varsigma(r) \mathrm{d} r+\frac{e}{e-1} q_{4} h \int_{t-h}^{t} W(x(r)) \mathrm{d} r+\frac{\left(1+\epsilon_{0}\right) e}{e-1} q_{5} h \int_{t-h}^{t} \sum_{l=1}^{n} \mu_{l}(r) \mathrm{d} r+d_{*}(t) \\
& \leq e^{-1} \varsigma(t-h)+\left[(n-1) k^{2} \beta+q_{5} \frac{\left(1+\epsilon_{0}\right) \beta}{1-\beta}\left(\beta^{-n}-1\right)\right] \frac{e h}{e-1} \int_{t-h}^{t} \varsigma(r) \mathrm{d} r+\frac{e q_{4} h}{e-1} \int_{t-h}^{t} W(x(r)) \mathrm{d} r+d_{*}(t),
\end{aligned}
$$

where $q_{5}=n q_{1}^{2} q_{3}$ and $d_{*}(t)=\bar{d} \int_{t-h}^{t} d^{2}(r) \mathrm{d} r$, the second inequality used the consequence $\left(\rho_{1}(r)+\ldots+\rho_{n}(r)\right)^{2} \leq$ $n\left(\rho_{1}^{2}(r)+\ldots+\rho_{n}^{2}(r)\right)=n\left(\mu_{1}(r)+\ldots \mu_{n}(r)\right)$ of Chebyshev's inequality, and the last inequality used the bound $\mu_{l}(r) \leq \beta^{l-n} \varsigma(r)$ for all $l$ and $h$ and all $r \geq 0$ and then the geometric sum formula. Let $q_{6}=\frac{e}{e-1} q_{4}$. Then

$$
\begin{aligned}
\varsigma(t) & \leq e^{-1} \varsigma(t-h)+\frac{e}{e-1}\left[(n-1) \beta+\frac{\left(1+\epsilon_{0}\right) q_{5} \beta}{1-\beta}\left(\beta^{-n}-1\right) h^{2}\right] \frac{1}{h} \int_{t-h}^{t} \varsigma(r) \mathrm{d} r+q_{6} h \int_{t-h}^{t} W(x(r)) \mathrm{d} r+d_{*}(t) \\
& \leq e^{-1} \varsigma(t-h)+\frac{e^{-1}\left(1-\epsilon_{0}\right)}{2 h} \int_{t-h}^{t} \varsigma(r) \mathrm{d} r+q_{6} h \int_{t-h}^{t} W(x(r)) \mathrm{d} r+d_{*}(t),
\end{aligned}
$$


since $k=1 / h$, by choosing $\epsilon_{0}>0$ small enough and using our assumption that $k>\bar{k}_{a}$ to check that our choices $q_{5}=n q_{1}^{2} q_{3}$ and $h=1 / k$ give

$$
\frac{e}{e-1}\left[(n-1) \beta+\frac{q_{5} \beta}{1-\beta}\left(\beta^{-n}-1\right) h^{2}\right]<\frac{1}{2 e} .
$$

Since $\sum_{l=1}^{n} \rho_{l}(t)=\lambda_{1}(t)-\psi(t)$, and since $\rho_{l}^{2}=\mu_{l}$ for all $l$, it follows from Assumption 4 (and from reapplying Chebyshev's inequality, as before) that along all solutions of (19), we have

$$
\dot{V}(t) \leq-W(x(t))+\mathcal{L}\left|\sum_{l=1}^{n} \rho_{l}(t)+d(t)\right|^{2} \leq-W(x(t))+\mathcal{L} q_{7} \sum_{l=1}^{n} \mu_{l}(t)+\left(1+\frac{1}{\epsilon_{0}}\right) \mathcal{L} d^{2}(t),
$$

where $q_{7}=\left(1+\epsilon_{0}\right) n$. Using the lower bounds $\varsigma(t) \geq \beta^{n-j} \mu_{j}(t)$, it follows from the geometric sum formula that

$$
\dot{V}(t) \leq-W(x(t))+\mathcal{L} q_{7} \sum_{l=1}^{n} \beta^{l-n} \varsigma(t)+\mathcal{L}^{\sharp} d^{2}(t)=-W(x(t))+\mathcal{L} q_{7} \frac{\beta^{-n}-1}{\beta^{-1}-1} \varsigma(t)+\mathcal{L}^{\sharp} d^{2}(t),
$$

where $\mathcal{L}^{\sharp}=\left(1+\left(1 / \epsilon_{0}\right)\right) \mathcal{L}$. Let $b_{2}=\sqrt{e}-1$. Then our choice of $\kappa\left(\varsigma_{t}\right)$ in $(29)$ gives $\dot{\kappa}(t)=-b_{1} \kappa\left(\varsigma_{t}\right)-b_{2} \varsigma(t)+$ $\left(1+b_{2}\right) \varsigma(t)-e^{-b_{1} h} \varsigma(t-h)$. Using $(31)$ and our choice $b_{1}=\frac{1}{2 h}$, we deduce that

$$
\begin{aligned}
\dot{\kappa}(t) \leq & -b_{1} \kappa\left(\varsigma_{t}\right)-b_{2} \varsigma(t)+\left(1+b_{2}\right)\left[e^{-1} \varsigma(t-h)+\frac{e^{-1}\left(1-\epsilon_{0}\right)}{2 h} \int_{t-h}^{t} \varsigma(r) \mathrm{d} r+q_{6} h \int_{t-h}^{t} W(x(r)) \mathrm{d} r\right] \\
& -e^{-b_{1} h} \varsigma(t-h)+\sqrt{e} d_{*}(t) \\
= & -b_{1} \int_{t-h}^{t} e^{-b_{1}(t-r)} \varsigma(r) \mathrm{d} r-b_{2} \varsigma(t)+\frac{\left(1+b_{2}\right) e^{-1}\left(1-\epsilon_{0}\right)}{2 h} \int_{t-h}^{t} \varsigma(r) \mathrm{d} r \\
& +\left(1+b_{2}\right) q_{6} h \int_{t-h}^{t} W(x(r)) \mathrm{d} r+\sqrt{e} d_{*}(t) \\
\leq & -\frac{1}{2 h} \int_{t-h}^{t} e^{-\frac{1}{2 h}(t-r)} \varsigma(r) \mathrm{d} r-b_{2} \varsigma(t)+\frac{1-\epsilon_{0}}{2 h} \int_{t-h}^{t} e^{-\frac{1}{2 h}(t-r)} \varsigma(r) \mathrm{d} r \\
& +q_{6} \sqrt{e} h \int_{t-h}^{t} W(x(r)) \mathrm{d} r+\sqrt{e} d_{*}(t) \\
= & -\frac{\epsilon_{0}}{2 h} \int_{t-h}^{t} e^{-\frac{1}{2 h}(t-r)} \varsigma(r) \mathrm{d} r-b_{2} \varsigma(t)+q_{6} \sqrt{e} h \int_{t-h}^{t} W(x(r)) \mathrm{d} r+\sqrt{e} d_{*}(t),
\end{aligned}
$$

where we also used the fact that $\left(1+b_{2}\right) e^{-1}-e^{-b_{1} h}=0$ and our definitions of $\kappa$ and $b_{1}$ in (29).

Let

$$
U\left(x_{t}, \varsigma_{t}\right)=V(x(t))+\frac{\left(1+\epsilon_{0}\right) \mathcal{L} q_{7}\left(\beta^{-n}-1\right)}{b_{2}\left(\beta^{-1}-1\right)} \kappa\left(\varsigma_{t}\right)+\frac{\left(1+\epsilon_{0}\right) q_{8}\left(\beta^{-n}-1\right)}{b_{2}\left(\beta^{-1}-1\right)} h \int_{t-h}^{t} \int_{m}^{t} W(x(r)) \mathrm{d} r \mathrm{~d} m,
$$

where $q_{8}=\mathcal{L} q_{6} q_{7} \sqrt{e}$. Then for a suitable constant $\bar{c}>0$, we can combine (34) with (35) to obtain

$$
\dot{U}(t) \leq-W(x(t))-\frac{\mathcal{L} q_{7} \epsilon_{0}\left(\beta^{-n}-1\right)}{\beta^{-1}-1} \varsigma(t)+\frac{q_{8}\left(1+\epsilon_{0}\right)\left(\beta^{-n}-1\right)}{b_{2}\left(\beta^{-1}-1\right)} h^{2} W(x(t))+\bar{c} d_{* *}(t),
$$

where $d_{* *}=\mathcal{L} d^{2}+d_{*}$. Consequently, since our choice of $\bar{k}_{b}$ from our assumption on $k=\frac{1}{h}$ implies that

$$
h<\sqrt{\frac{b_{2}\left(\beta^{-1}-1\right)}{q_{8}\left(\beta^{-n}-1\right)\left(1+\epsilon_{0}\right)}}
$$

when $\epsilon_{0}>0$ is small enough, we can find a constant $c_{*}>0$ such that

$$
\dot{U}(t) \leq-c_{*} W(x(t))-\frac{\epsilon_{0} \mathcal{L} q_{7}\left(\beta^{-n}-1\right)}{\beta^{-1}-1} \varsigma(t)+\bar{c} d_{* *}(t) .
$$

Also, since $d(t)$ exponentially decays to zero, we have $\int_{0}^{\infty} \int_{s-h}^{s} d^{2}(r) \mathrm{d} r \mathrm{~d} s<+\infty$ and $\int_{0}^{\infty} d^{2}(r) \mathrm{d} r<+\infty$. Since $W$ is uniformly continuous, the lemma follows from Barbalat's Lemma, by integrating (39) over $[0,+\infty)$ (which implies that $x(t)$ is bounded, so $(x(t), \lambda(t))$ is bounded, by the boundedness of $\psi(t, x(t)))$, then concluding that $\lim _{t \rightarrow+\infty} x(t)=\lim _{t \rightarrow+\infty} \varsigma(t)=0$, then using the definition of $\varsigma$ to also conclude that $\lim _{t \rightarrow+\infty} \rho_{i}(t)=0$ and therefore also $\lim _{t \rightarrow+\infty} \lambda_{i}(t)=0$ for each $i$ (because Assumption 3 now implies that $\left.\lim _{t \rightarrow+\infty} \psi(t, x(t))=0\right)$.

Remark 3.1. The first condition in Assumption 3 is the requirement of a uniform global Lipschitz constant $q_{1}$ for $\psi$. We can prove a variant of the preceding lemma if we replace this uniform global Lipschitz requirement by the assumptions that (i) $\psi$ is uniformly locally Lipschitz in the state uniformly in time (meaning, for each constant $R>0$, there is a constant $\bar{q}_{R}>0$ such that $|\psi(t, a)-\psi(t, b)| \leq \bar{q}_{R}|a-b|$ holds for all $a$ and $b$ that satisfy $\max \{|a|,|b|\} \leq R$ and all $t \geq 0$ ), (ii) $\psi$ is bounded, and (iii) $W$ in Assumption 4 is proper. Condition 
(ii) is not at all restrictive, because $\psi$ is assumed to be bounded in our Assumption 1 in the statement of our Theorem 1. With the preceding replacements in Assumptions 3-5, we can now prove a semiglobal (instead of a global) version of Lemma 1 on a sequence of sets whose nested union is our entire state space $\mathbb{R}^{n+p}$, using the following algorithm: Step 1: Given any constant $r>0$, use the input-to-state stability Lyapunov function decay condition on $V$ from Assumption 4 (and standard input-to-state stability arguments from [12, Chapter 4]) to find a constant $R>0$ such that $\sup _{t \geq 0}|x(t)| \leq R$ holds for each solution $x(t)$ of $\dot{x}(t)=f(t, x(t), \psi(t, x(t))+\delta(t))$ whose its initial state satisfies $\left|x\left(t_{0}\right)\right| \leq r$ and each continuous function $\delta$ that is bounded by $2 \bar{\psi}+|d|_{\infty}+1$, namely, $R=\beta_{0}(r, 0)+\gamma_{0}\left(2 \bar{\psi}+|d|_{\infty}+1\right)$ where the class $\mathcal{K} \mathcal{L}$ function $\beta_{0}$ and the class $\mathcal{K}_{\infty}$ function $\gamma_{0}$ are the comparison functions in the input-to-state stability estimate. Step 2: Choose constants $k>0$ and $\beta \in(0,1)$ such that $(21)$ is satisfied with $q_{1}$ replaced by $\bar{q}_{R}$ (so $k$ and $\beta$ will now depend on $R$ ). Then the same argument we used to prove Lemma 1 shows that with the choice of $k$ from Step 2, all solutions of $(x, \lambda):[0,+\infty) \rightarrow \mathbb{R}^{p+n}$ of (19) having initial states that satisfy $|x(0)| \leq r$ converge to 0 as $t \rightarrow+\infty$, except that instead of considering all times $t \geq h$ in the proof of Lemma 1 , we constrain the time argument $t$ in the proof to satisfy $t \geq \max \{h, T(|\lambda(0)|)\}$ where the function $T$ is such that $\left|\lambda_{1}(t)\right| \leq \bar{\psi}+1$ for all $t \geq T(|\lambda(0)|)$, and then we apply the input-to-state stability condition from Step 1 to the function $\delta(t)=-\psi(t, x(t))+\lambda_{1}(t)+d(t)$ (which satisfies $|\delta(t)| \leq 2 \bar{\psi}+|d|_{\infty}+1$ for all $t \geq T(|\lambda(0)|)$ ). Then we can use the semiglobal version of the lemma to find sufficient conditions for a semiglobal version of our theorem, by allowing the $k$ in Assumption 1, in (6a)-(6d), and in the control (7) to depend on an upper bound on the norm of the initial state. We leave the remaining details to the reader.

\section{Illustrations}

Theorem 1 applies to a broad class of nonlinear systems, without requiring time lagged values of the state in the feedback. In this section, we illustrate these points in two examples. In our first example, we apply Lemma 1 to check our assumptions from Theorem 1. In our second example, we use an alternative method to check the assumptions, to cope with the presence of multiple unknown parameters in the system.

\subsection{First Illustration}

We apply Lemma 1 to the weakly nonminimum phase dynamics

$$
\left\{\begin{aligned}
\dot{x}(t) & =\omega \Lambda(x(t))+g_{1}(t) \\
\dot{g}_{1}(t) & =g_{2}(t) \\
\dot{g}_{2}(t) & =u(t)
\end{aligned}\right.
$$

with the state space $\mathbb{R}^{3}$ where $\omega>0$ is a constant and $\Lambda$ is a globally Lipschitz function having global Lipschitz constant 1 , which is not amenable to classical backstepping, because the right side of $\dot{x}(t)$ will not in general be differentiable. In [21], we used an artificial delay approach to construct a globally asymptotically stabilizing bounded backstepping controller for (42) in the special case where $\omega=1$ and $\Lambda(x)=|x| /(1+|x|)$, which required the current and one time lagged value of the state in the feedback. On the other hand, we can satisfy the requirements from Lemma 1 for (42) with $n=2, a_{1,1}=a_{2,1}=a_{2,2}=0, a_{1,2}=1$, and $\Omega=0$, using

$$
f\left(t, x, g_{1}\right)=\omega \Lambda(x)+g_{1}, \psi(x)=-\omega \Lambda(x)-\omega \operatorname{sat}_{1}(x), V(x)=\frac{1}{\omega} \int_{0}^{x} \operatorname{sat}_{1}(\ell) \mathrm{d} \ell, \text { and } W(x)=\frac{1}{2} \operatorname{sat}_{1}^{2}(x),
$$

since along all solutions of $\dot{x}(t)=f(x(t), \psi(x)+\delta(t))$ for all $t \geq 0$, these choices and the triangle inequality give

$$
\dot{V}(t)=-\operatorname{sat}_{1}^{2}(x)+\frac{1}{\omega} \operatorname{sat}_{1}(x) \delta(t) \leq-\frac{1}{2} \operatorname{sat}_{1}^{2}(x)+\frac{1}{2 \omega^{2}} \delta^{2}(t),
$$

and we can choose $\mathcal{L}=\frac{1}{2 \omega^{2}}, q_{1}=2 \omega, q_{2}=6 \omega^{2}$, and $q_{3}=3 / 2$ (by the inequality $\left(-\omega \operatorname{sat}_{1}(x)+\Delta\right)^{2} \leq$ $\left.3 \omega^{2} \operatorname{sat}_{1}^{2}(x)+(3 / 2) \Delta^{2}\right)$. We can also choose $\beta=\frac{e-1}{4.1 e^{2}}$, and then $\bar{k}_{a}$ and $\bar{k}_{b}$ from Lemma 1 with the preceding choices are

$$
\bar{k}_{a}=2 \omega e \sqrt{\frac{6 \beta\left(\beta^{-2}-1\right)}{\left(e-1-2 e^{2} \beta\right)(1-\beta)}} \text { and } \bar{k}_{b}=2 \omega \sqrt{\frac{6 e^{3 / 2}\left(\beta^{-2}-1\right)}{(e-1)(\sqrt{e}-1)\left(\beta^{-1}-1\right)}}
$$

so Assumption 1 holds for any $k>\max \left\{\bar{k}_{a}, \bar{k}_{b}\right\}=61.2729 \omega$. Also, Assumption 2 holds by well known nested saturation controls for chains of integrators. Hence, the desired feedback control is provided by Theorem 1. 

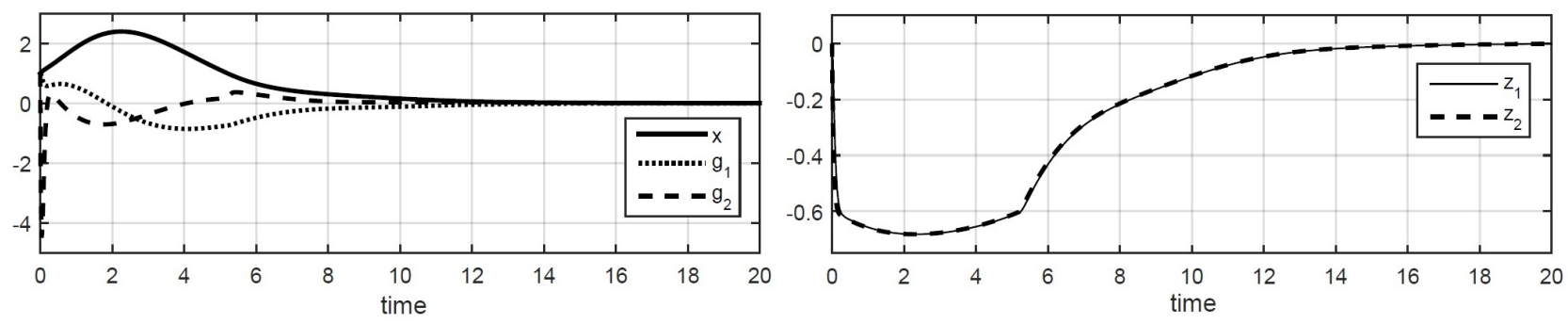

Figure 1: Feedback Control in First Illustration

In Fig. 1, we plot MATLAB simulations for the preceding system in closed loop with the control provided by our theorem, with $\epsilon=0.05, \omega=0.4$, and $\Lambda(x)=|x| /(1+|x|)$. The figure shows the good performance of our control, and therefore helps illustrate the value of our theorem in a special case of the dynamics (42).

\subsection{Second Illustration}

We consider the dynamics

$$
\left\{\begin{array}{l}
\dot{x}_{1}=x_{2} \\
\dot{x}_{2}=p_{0}\left(g_{1}-p_{1} \sin \left(x_{1}\right)-p_{2} x_{2}\right) \\
\dot{g}_{1}=u-p_{3} \arctan \left(x_{2}\right)+a_{1,1} g_{1}
\end{array}\right.
$$

with the output $Y=\left(x, g_{1}\right)$ and the $\mathbb{R}^{2}$-valued state components $x=\left(x_{1}, x_{2}\right)$, where $a_{1,1}<0$ and $p_{i}>0$ for $i=0$ to 3 are constants. This includes the dynamics of a single-link direct-drive manipulator actuated by a permanent magnet DC brush motor from [4], which are (46) in the special case where $p_{0}=1$ (after a change of coordinates which removes a constant). Assumption 2 is satisfied with $\varpi=0$ and $n=1$ (because in this case, (5) with $\varpi=0$ is a linear system that is exponentially stable to 0$)$, and we choose $\Omega\left(x, g_{1}\right)=-p_{3} \arctan \left(x_{2}\right)$. To illustrate how Theorem 1 covers cases where the nonlinear part of the dynamics may not be known, let us assume that $a_{1,1}, p_{1}$, and $p_{3}$ are known, but that $p_{0}$ and $p_{2}$ are not known (so we may use $a_{1,1}, p_{1}$, and $p_{3}$ in the control, but we cannot use $p_{0}$ or $p_{2}$ in the control). The uncertainty of $p_{0}$ (resp., $p_{2}$ ) can represent an uncertain mass (resp., viscous friction coefficient) in the manipulator dynamics. However, we assume that

$\frac{2 p_{1}}{1+p_{1}}<p_{2}<\frac{1+p_{1}}{p_{1}}$ and $\left|p_{0}-1\right|<\min \left\{\bar{p}_{1}, \bar{p}_{2}\right\}$, where $\bar{p}_{1}=\frac{3\left(1+p_{1}\right) p_{1}}{2 p_{1}^{2}+8 p_{1}\left(1+p_{1}\right)+16\left(1+p_{1}\right)^{2}}$ and $\bar{p}_{2}=\frac{p_{2}}{4 p_{2}^{2}+8 p_{2}+8}$

and we choose any constant $k>0$ such that

$$
k>\frac{8}{5}\left(\frac{1}{2}\left(\frac{1}{p_{2}}+\frac{p_{1}}{1+p_{1}}\right)+2\left|p_{0}-1\right|\right) \text { and } k>\frac{16}{3 p_{2}}\left(p_{1}+1\right)^{2} .
$$

We will show that (47)-(48) ensure that Assumption 1 is satisfied with $n=1$ for any $k$ that satisfies (48).

Consider

$$
\psi(\xi)=p_{1} \sin \left(\xi_{1}\right)-\left\langle\xi_{1}\right\rangle, \text { where }\left\langle\xi_{1}\right\rangle=\frac{\xi_{1}}{\sqrt{1+\xi_{1}^{2}}}
$$

and any continuous function $d:[0,+\infty) \rightarrow \mathbb{R}$ that exponentially converges to 0 and

$$
\dot{\xi}_{1}=\xi_{2}, \quad \dot{\xi}_{2}=p_{0}\left(-p_{1} \sin \left(\xi_{1}\right)-p_{2} \xi_{2}+\lambda_{1}+d(t)\right), \quad \dot{\lambda}_{1}=k\left[-\lambda_{1}+p_{1} \sin \left(\xi_{1}\right)-\left\langle\xi_{1}\right\rangle\right] .
$$

Let us choose

$$
V(\xi)=\sqrt{1+\xi_{1}^{2}}-1+\frac{1}{2} \xi_{2}^{2}+\frac{p_{1}}{4\left(1+p_{1}\right)}\left\langle\xi_{1}\right\rangle \xi_{2} \text { and } s_{1}=\lambda_{1}-p_{1} \sin \left(\xi_{1}\right)+\left\langle\xi_{1}\right\rangle .
$$

In the new variables $\left(\xi, s_{1}\right)$, the system $(50)$ becomes

$$
\dot{\xi}_{1}=\xi_{2}, \quad \dot{\xi}_{2}=-p_{2} \xi_{2}-\left\langle\xi_{1}\right\rangle+s_{1}+d(t)+\left(p_{0}-1\right) \mathcal{M}(t), \quad \dot{s}_{1}=-k s_{1}+\left[-p_{1} \cos \left(\xi_{1}\right)+\frac{1}{\left(1+\xi_{1}^{2}\right) \sqrt{1+\xi_{1}^{2}}}\right] \xi_{2},
$$

where $\mathcal{M}(t)=-p_{2} \xi_{2}-\left\langle\xi_{1}\right\rangle+s_{1}+d(t)$ and where we omit the time argument of the state variables to simplify the notation. Also, the second inequality in (47) and the triangle inequality combine to give

$$
-p_{2} \frac{p_{1}}{4\left(1+p_{1}\right)} \frac{\xi_{1} \xi_{2}}{\sqrt{1+\xi_{1}^{2}}} \leq \frac{p_{1}}{4\left(1+p_{1}\right)}\left(\frac{1}{2} \frac{\xi_{1}^{2}}{1+\xi_{1}^{2}}+\frac{1}{2} p_{2}^{2} \xi_{2}^{2}\right) \leq \frac{p_{1}}{8\left(1+p_{1}\right)} \frac{\xi_{1}^{2}}{1+\xi_{1}^{2}}+\frac{p_{2}}{8} \xi_{2}^{2} .
$$


Therefore, for any constant $\epsilon_{0} \in(0,1)$, and with the choice $\mathcal{M}^{\sharp}(t)=\left(p_{0}-1\right) \frac{\partial V(\xi)}{\partial \xi_{2}}(t) \mathcal{M}(t)$, we obtain

$$
\begin{aligned}
\dot{V}(t)= & \frac{\partial V(\xi)}{\partial \xi_{1}} \xi_{2}+p_{0} \frac{\partial V(\xi)}{\partial \xi_{2}}\left(-p_{2} \xi_{2}-\left\langle\xi_{1}\right\rangle+s_{1}+d(t)\right) \\
= & -p_{2}\left(\xi_{2}^{2}+\frac{p_{1}}{4\left(1+p_{1}\right)} \frac{\xi_{1} \xi_{2}}{\sqrt{1+\xi_{1}^{2}}}\right)+\mathcal{M}^{\sharp}(t) \\
& -\frac{p_{1}}{4\left(1+p_{1}\right)} \frac{\xi_{1}^{2}}{1+\xi_{1}^{2}}+\frac{p_{1}}{4\left(1+p_{1}\right)} \frac{\xi_{2}^{2}}{\left(1+\xi_{1}^{2}\right) \sqrt{1+\xi_{1}^{2}}}+\left(\xi_{2}+\frac{p_{1}}{4\left(1+p_{1}\right)}\left\langle\xi_{1}\right\rangle\right)\left(s_{1}+d(t)\right) \\
\leq & -\frac{3 p_{2}}{4} \xi_{2}^{2}-\frac{p_{1}}{8\left(1+p_{1}\right)} \frac{\xi_{1}^{2}}{1+\xi_{1}^{2}}+\xi_{2}\left(s_{1}+d(t)\right)+\frac{p_{1}}{4\left(1+p_{1}\right)}\left\langle\xi_{1}\right\rangle\left(s_{1}+d(t)\right)+\mathcal{M}^{\sharp}(t) \\
\leq & -\frac{p_{2}}{4} \xi_{2}^{2}-\frac{3 p_{1}}{32\left(1+p_{1}\right)} \frac{\xi_{1}^{2}}{1+\xi_{1}^{2}}+\frac{1+\epsilon_{0}}{2}\left(\frac{1}{p_{2}}+\frac{p_{1}}{1+p_{1}}\right) s_{1}^{2}+\frac{1}{2}\left(1+\frac{1}{\epsilon_{0}}\right)\left(\frac{1}{p_{2}}+\frac{p_{1}}{1+p_{1}}\right) d^{2}(t)+\mathcal{M}^{\sharp}(t)
\end{aligned}
$$

along all solutions of (52) for all $t \geq 0$, where the first inequality in (54) used the first inequality in (47) and (53), and the second inequality in (54) used the relations

$$
\xi_{2}\left(s_{1}+d(t)\right) \leq \frac{1}{2} p_{2} \xi_{2}^{2}+\frac{1}{2 p_{2}}\left(s_{1}+d(t)\right)^{2} \text { and }\left\langle\xi_{1}\right\rangle\left(s_{1}+d(t)\right) \leq \frac{1}{8} \frac{\xi_{1}^{2}}{1+\xi_{1}^{2}}+2\left(s_{1}+d(t)\right)^{2},
$$

followed by the inequality $\left(s_{1}+d(t)\right)^{2} \leq\left(1+\epsilon_{0}\right) s_{1}^{2}+\left(1+\left(1 / \epsilon_{0}\right)\right) d^{2}(t)$. Also, since

$$
\sqrt{1+p}-1 \geq \frac{p}{2(1+p)} \text { for all } p \geq 0,
$$

it follows from (53) with $p_{2}$ replaced by 1 that the choice of $V$ in (51) is proper and positive definite.

Next let $U\left(\xi, s_{1}\right)=V(\xi)+\frac{1}{2} s_{1}^{2}$. Then $(54)$ gives

$$
\begin{aligned}
\dot{U}(t) \leq & -\frac{p_{2}}{4} \xi_{2}^{2}-\frac{3 p_{1}}{32\left(1+p_{1}\right)} \frac{\xi_{1}^{2}}{1+\xi_{1}^{2}}+\frac{1+\epsilon_{0}}{2}\left(\frac{1}{p_{2}}+\frac{p_{1}}{1+p_{1}}\right) s_{1}^{2}+\frac{1}{2}\left(1+\frac{1}{\epsilon_{0}}\right)\left(\frac{1}{p_{2}}+\frac{p_{1}}{1+p_{1}}\right) d^{2}(t)-k s_{1}^{2} \\
& +\left[-p_{1} \cos \left(\xi_{1}\right)+\frac{1}{\left(1+\xi_{1}^{2}\right) \sqrt{1+\xi_{1}^{2}}}\right] s_{1} \xi_{2}+\mathcal{M}^{\sharp}(t) \\
\leq & -\frac{p_{2}}{4} \xi_{2}^{2}-\frac{3 p_{1}}{32\left(1+p_{1}\right)} \frac{\xi_{1}^{2}}{1+\xi_{1}^{2}}+\left(p_{1}+1\right)\left|s_{1} \xi_{2}\right|+\left[\frac{1+\epsilon_{0}}{2}\left(\frac{1}{p_{2}}+\frac{p_{1}}{1+p_{1}}\right)-k\right] s_{1}^{2} \\
& +\frac{1}{2}\left(1+\frac{1}{\epsilon_{0}}\right)\left(\frac{1}{p_{2}}+1\right) d^{2}(t)+\mathcal{M}^{\sharp}(t)
\end{aligned}
$$

along all solutions of (50) for all $t \geq 0$. Since $\left(p_{1}+1\right)\left|s_{1} \xi_{2}\right| \leq \frac{3 k}{8} s_{1}^{2}+\frac{2}{3 k}\left(p_{1}+1\right)^{2} \xi_{2}^{2}$, it follows from our conditions on $\left|p_{0}-1\right|$ and $k$ from (47)-(48) that if we choose $\epsilon_{0} \in(0,1)$ close enough to 0 , then we can find a constant $c_{0}>0$ such that

$$
\begin{aligned}
\dot{U}(t) \leq & -\frac{p_{2}}{4} \xi_{2}^{2}-\frac{3 p_{1}}{32\left(1+p_{1}\right)} \frac{\xi_{1}^{2}}{1+\xi_{1}^{2}}+\frac{2}{3 k}\left(p_{1}+1\right)^{2} \xi_{2}^{2}+\left[\frac{1+\epsilon_{0}}{2}\left(\frac{1}{p_{2}}+\frac{p_{1}}{1+p_{1}}\right)-\frac{5}{8} k\right] s_{1}^{2} \\
& +\frac{1}{2}\left(1+\frac{1}{\epsilon_{0}}\right)\left(\frac{1}{p_{2}}+1\right) d^{2}(t)+\mathcal{M}^{\sharp}(t) \\
\leq & -c_{0}\left\{\xi_{2}^{2}+\frac{\xi_{1}^{2}}{1+\xi_{1}^{2}}+s_{1}^{2}\right\}+\left(\frac{1}{2}\left(1+\frac{1}{\epsilon_{0}}\right)\left(\frac{1}{p_{2}}+1\right)+2\left|p_{0}-1\right|\right) d^{2}(t)
\end{aligned}
$$

along all solutions of (50) for all $t \geq 0$, because the second inequality in (48) implies that $\frac{2}{3 k}\left(p_{1}+1\right)^{2} \leq \frac{p_{2}}{8}$, and because our bounds $\bar{p}_{1}$ and $\bar{p}_{2}$ on $\left|p_{0}-1\right|$ from (47) imply that there are constants $c_{a} \in\left(0, p_{2} / 8\right)$ and $c_{b} \in\left(0,3 p_{1} /\left(32\left(1+p_{1}\right)\right)\right.$ such that

$$
\begin{aligned}
\mathcal{M}^{\sharp}(t)= & \left(p_{0}-1\right)\left(\xi_{2}+\frac{p_{1}}{4\left(1+p_{1}\right)}\left\langle\xi_{1}\right\rangle\right)\left(-p_{2} \xi_{2}-\left\langle\xi_{1}\right\rangle+s_{1}+d(t)\right) \\
\leq & \left|p_{0}-1\right|\left(p_{2} \xi_{2}^{2}+\left\{\frac{1}{2} \xi_{2}^{2}+\frac{1}{2} \frac{\xi_{1}^{2}}{1+\xi_{1}^{2}}\right\}+\left\{\frac{1}{2} \xi_{2}^{2}+s_{1}^{2}+d^{2}(t)\right\}\right. \\
& \left.+\left\{\frac{1}{2}\left(\frac{p_{1}}{4\left(1+p_{1}\right)}\right)^{2} \frac{\xi_{1}^{2}}{1+\xi_{1}^{2}}+\frac{1}{2} p_{2}^{2} \xi_{2}^{2}\right\}+\frac{p_{1}}{4\left(1+p_{1}\right)} \frac{\xi_{1}^{2}}{1+\xi_{1}^{2}}+\left\{\frac{1}{2}\left(\frac{p_{1}}{4\left(1+p_{1}\right)}\right)^{2} \frac{\xi_{1}^{2}}{1+\xi_{1}^{2}}+s_{1}^{2}+d^{2}(t)\right\}\right) \\
\leq & c_{a} \xi_{2}^{2}+c_{b} \frac{\xi_{1}^{2}}{1+\xi_{1}^{2}}+2\left|p_{0}-1\right|\left(s_{1}^{2}+d^{2}(t)\right)
\end{aligned}
$$

for all $t \geq 0$, by repeated applications of the triangle inequality; the first set of terms in curly braces in (58) were obtained from using the triangle inequality to upper bound $\left|\xi_{2}\left\langle\xi_{1}\right\rangle\right|$, the second set of terms in curly braces in (58) were used to upper bound $\left|\xi_{2}\left(s_{1}+d(t)\right)\right|$, and similarly for the other terms in curly braces in (58). It 
follows from integrating (57) over $[0,+\infty)$ and applying Barbalat's Lemma to the function in curly braces in (57) that Assumption 1 is satisfied (because $d^{2}(t)$ exponentially converges to 0 as $t \rightarrow+\infty$ ). Hence, Theorem 1 applies to (46) and provides the dynamic feedback

$$
u(Z, x)=-\operatorname{sat}_{\bar{Z}}\left(\left(a_{1,1}+k\right) z_{1}-k \psi\left(x_{1}\right)\right)+p_{3} \arctan \left(x_{2}\right), \quad \dot{z}_{1}=k\left[-z_{1}+p_{1} \sin \left(x_{1}\right)-\left\langle x_{1}\right\rangle\right]
$$

with $\bar{Z}=(1+\epsilon)\left(\left|a_{1,1}+k\right|+k\right)\left(p_{1}+1\right)$ and the choice (49) of $\psi$, since our formulas (6a)-(6b) give $c_{1}=a_{1,1}+k$.

In Fig. 2, we plot MATLAB simulations for the system (46) in closed loop with the control provided by our theorem, with $\epsilon=0.05, p_{0}=p_{1}=p_{3}=1, p_{2}=1.5$, and $a_{1,1}=-1$. The figure shows the good performance of our control, and therefore helps illustrate the value of our theorem in a specific case of the dynamics (46). Moreover, with the choice $p_{1}=1$, our conditions (47) imply that our control is also valid for any constants $p_{2} \in(1,2)$ and $p_{0} \in(0.95,1.05)$, which means that the viscous friction coefficient (resp., mass) measurement can tolerate a $50 \%$ (resp., $5 \%$ ) error. This illustrates the robustness of our control design to uncertainties.
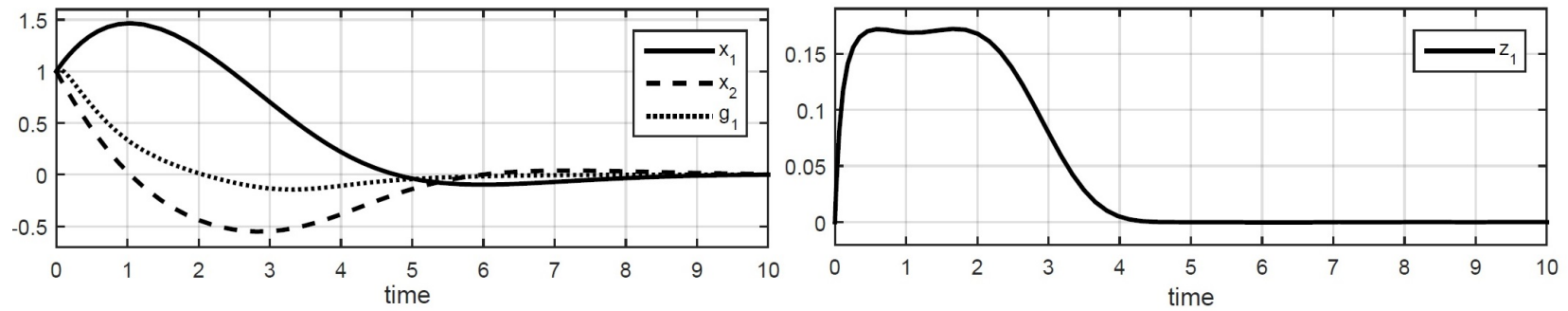

Figure 2: Feedback Control in Second Illustration

\section{Conclusions}

We provided a new backstepping approach using a finite dimensional dynamic extension, which is motivated by the ubiquity of engineering applications that lead to the required cascade forms. Advantages of our approach include the facts that we do not require the fictitious feedback to be $C^{1}$, and the fact that we can satisfy input bounds without using artificial delays. We hope to develop local versions for systems that are only locally asymptotically stabilizable, and to allow measurement delays [29, 31]. We will also investigate families of systems that can be stabilized by applying Theorem 1 repeatedly, and possible extensions to PDEs.

\section{References}

[1] B. Bialy, J. Klotz, J. Curtis, and W. Dixon. An adaptive backstepping controller for a hypersonic airbreathing missile. In Proceedings of the AIAA Guidance Navigation and Control Conference. Minneapolis, MN, Paper AIAA2012-4468, 2012.

[2] J. Chen, W. Dixon, J. Wagner, and D. Dawson. Exponential tracking control of a hydraulic proportional directional valve and cylinder via integrator backstepping. In Proceedings of 2002 ASME International Mechanical Engineering Congress and Exposition. New Orleans, LA, Paper IMECE2002-32076, 2002.

[3] J-M. Coron and L. Praly. Adding an integrator for the stabilization problem. Systems and Control Letters, 17:89-104, 1991.

[4] D. Dawson, J. Carroll, and M. Schneider. Integrator backstepping control of a brush DC motor turning a robotic load. IEEE Transactions on Control Systems Technology, 2:233-244, 1994.

[5] W. Dixon, Z-P. Jiang, and D. Dawson. Global exponential setpoint control of wheeled mobile robots: a Lyapunov approach. Automatica, 36:1741-1746, 2000.

[6] J. Farrell, M. Polycarpou, M. Sharma, and W. Dong. Command filtered backstepping. IEEE Transactions on Automatic Control, 54(6):1391-1395, 2009.

[7] T. Hu. A nonlinear-system approach to analysis and design of power-electronic converters with saturation and bilinear terms. IEEE Transactions on Power Electronics, 26(2):399-410, 2011.

[8] T. Hu and Z. Lin. Control Systems with Actuator Saturation. Birkhauser, Boston, 2001.

[9] A Iovine, S. Siad, G. Damm, E. De Santis, and M. Di Benedetto. Nonlinear control of a DC microgrid for the integration of photovoltaic panels. IEEE Transactions on Automation Science and Engineering, 14(2):524-535, 2017. 
[10] A. Isidori. Nonlinear Control Systems. Springer-Verlag, New York, 1995.

[11] Z-P. Jiang and H. Nijmeijer. A recursive technique for tracking control of nonholonomic systems in chained form. IEEE Transactions on Automatic Control, 44:265-279, 1999.

[12] H. Khalil. Nonlinear Systems. Prentice Hall, Upper Saddle River, NJ, 2002.

[13] D. Lee, C. Nataraj, T. Burg, and D. Dawson. Adaptive tracking control of an underactuated aerial vehicle. In Proceedings of the American Control Conference, pages 2326-2331. San Francisco, CA, 2011.

[14] Y. Li and Z. Lin. Stability and Performance of Control Systems with Actuator Saturation. Birkhauser, Boston, 2018.

[15] F. Mazenc and S. Bowong. Backstepping with bounded feedbacks for time-varying systems. SIAM Journal on Control and Optimization, 43:856-871, 2004.

[16] F. Mazenc, L. Burlion, and V. Gibert. Stabilization of a system that arises in the context of vision based landing of a civil aircraft. In Proceedings of the American Control Conference, pages 2978-2983. Milwaukee, WI, 2018.

[17] F. Mazenc, L. Burlion, and M. Malisoff. Backstepping design for output feedback stabilization for a class of uncertain systems using dynamic extension. In Proceedings of the 2nd IFAC Conference on Modelling, Identification, and Control of Nonlinear Systems, pages 260-265. Guadalajara, Mexico, 2018.

[18] F. Mazenc and A. Iggidr. Backstepping with bounded feedbacks. Systems and Control Letters, 51:235-245, 2004.

[19] F. Mazenc and M. Malisoff. New control design for bounded backstepping under input delay. Automatica, $6: 48-55,2016$.

[20] F. Mazenc, M. Malisoff, L. Burlion, and V. Gibert. Bounded backstepping through a dynamic extension with delay. In Proceedings of the 56th IEEE Conference on Decision and Control, pages 607-611. Melbourne, Australia, 2017.

[21] F. Mazenc, M. Malisoff, L. Burlion, and J. Weston. Bounded backstepping control and robustness analysis for time-varying systems under converging-input-converging-state conditions. European Journal of Control, 42:15-24, 2018.

[22] F. Mazenc, M. Malisoff, and J. Weston. New bounded backstepping control designs for time-varying systems under converging input converging state conditions. In Proceedings of the 55th IEEE Conference on Decision and Control, pages 3167-3171. Las Vegas, NV, 2016.

[23] K. Pettersen and H. Nijmeijer. Underactuated ship tracking control; theory and experiments. IEEE Transactions on Automatic Control, 40:1759-1762, 2002.

[24] A. Sezkin and M. Krstic. Boundary backstepping control of flow-induced vibrations of a membrane at high Mach numbers. ASME Journal of Dynamic Systems, Measurement, and Control, 137(8), Paper DS-141203, 2015.

[25] M. Smaoui, X. Brun, and D. Thomasset. A study on tracking position control of an electropneumatic system using backstepping design. Control Engineering Practice, 14:923-933, 2006.

[26] H. Sussmann, E. Sontag, and Y. Yang. A general result on the stabilization of linear systems using bounded controls. IEEE Transactions on Automatic Control, 39(12):2411-2425, 1994.

[27] S. Tarbouriech and J.M. Gomes da Silva. Synthesis of controllers for continuous-time delay systems with saturating controls via LMIs. IEEE Transactions on Automatic Control, 45(1):105-111, 2000.

[28] J. Tsinias. Input to state stability properties of nonlinear systems and applications to bounded feedback stabilization using saturation. ESAIM: Control, Optimisation and Calculus of Variations, 2:57-85, 1997.

[29] Y. Wei and Z. Lin. Stability criteria of linear systems with multiple input delays under truncated predictor feedback. Systems and Control Letters, 111:9-17, 2018.

[30] Y. Yin, Z. Lin, Y. Liu, and K. Teo. Event-triggered constrained control of positive systems with input saturation. International Journal of Robust and Nonlinear Control, 28(11):3532-3542, 2018.

[31] S. Yoon, L. Di, P. Anantachaisilp, and Z. Lin. Truncated predictor feedback control for active magnetic bearing systems with input delay. IEEE Transactions on Control Systems Technology, 24(6):2182-2189, 2016.

[32] Y. Zhu, M. Krstic, H. Su, and C. Xu. Linear backstepping output feedback control for uncertain linear systems. International Journal of Adaptive Control and Signal Processing, 30(8-10):1080-1098, 2016. 